Göteborg-ITP-93-33

hep-th/9310177

October, 1993

\title{
A Note on the Relation between Different Forms of Superparticle Dynamics
}

\author{
Martin CEDERWALl \\ Institute for Theoretical Physics \\ Chalmers University of Technology and University of Göteborg \\ S-412 96 Göteborg, Sweden
}

\begin{abstract}
A formulation of $D=10$ superparticle dynamics is given that contain space-time and twistor variables. The set of constraints is entirely first class, and gauge conditions may be imposed that reduces the system to a Casalbuoni-Brink-Schwarz superparticle, a spinning particle or a twistor particle.
\end{abstract}


In reference [1], Sorokin, Tkach, Volkov and Zheltukhin established the equivalence between the Casalbuoni-Brink-Schwarz (CBS) superparticle [2] and the spinning particle in $D=10$ [3]. Their formulation, that has led to progress in the understanding of the fermionic symmetries $[4,5]$ of supersymmetric particles [6,7], introduced a "twistor-like" variable $\lambda^{\alpha}$, a bosonic spinor. In this note, I will show that by making this spinor dynamical one can formulate a dynamical system that contains the CBS superparticle and the spinning particle as well as the division algebra twistor formulation of the superparticle [8,9] as special gauge choices. Unlike in reference [1], there is no manifest world-line supersymmetry. It is not impossible, though, that this constraint structure will arise from a manifest $N=8$ world-line superconformally symmetric treatment based on $S^{7}$ $[10,11,12]$. The present framework is rather reminiscent of that in reference [13].

The literature on the supersymmetric point particle and its (covariant) first quantization is vast. The list of references only contains items of immediate interest for the issues addresses here, and I apologize for leaving out many important contributions. The content of this note is by no means revolutionary, but it is probably the simplest way of establishing the equivalence of the different forms of superparticle dynamics.

The phase space variables are $\left(X^{\mu}, P^{\mu}\right)$, parametrizing Minkowski phase space, $\left(\vartheta^{\alpha}, p_{\alpha}\right)$, the fermionic variables (spinors) of the CBS superparticle, $\left(\lambda^{\alpha}, \omega_{\alpha}\right)$, the twistor variables, and $\xi^{\mu}$, the fermionic vector of the twistor particle or spinning particle. Their Poisson brackets are

$$
\begin{array}{ll}
\left\{X^{\mu}, P^{\nu}\right\}=\eta^{\mu \nu}, & \left\{\vartheta^{\alpha}, p_{\beta}\right\}=\delta_{\beta}^{\alpha}, \\
\left\{\lambda^{\alpha}, \omega_{\beta}\right\}=\delta_{\beta}^{\alpha}, & \left\{\xi^{\mu}, \xi^{\nu}\right\}=-\eta^{\mu \nu} .
\end{array}
$$

For this set of variables, I postulate the constraints

$$
\begin{aligned}
& \Pi^{\mu} \equiv P^{\mu}-\frac{1}{2}\left(\lambda \gamma^{\mu} \lambda\right) \approx 0, \\
& \pi_{\alpha} \equiv p_{\alpha}+P_{\mu}\left(\gamma^{\mu} \vartheta\right)_{\alpha}+\xi_{\mu}\left(\gamma^{\mu} \lambda\right)_{\alpha} \approx 0, \\
& T^{\alpha} \equiv \frac{1}{2}\left(\lambda \gamma_{\mu} \lambda\right)\left(\gamma^{\mu} \omega\right)^{\alpha}-\lambda^{\alpha}(\lambda \omega)-\frac{1}{2} \xi_{\mu} \xi_{\nu}\left(\gamma^{\mu \nu} \lambda\right)^{\alpha} \approx 0, \\
& t \equiv \frac{1}{2}\left(\lambda \gamma_{\mu} \lambda\right) \xi^{\mu} \approx 0 .
\end{aligned}
$$

The first constraint states the twistor transformation of the particle momentum, and implies its lightlikeness. The second one is the usual set of fermionic constraints for the superparticle, modified with the last term which makes it first class. The last two constraints are the $S^{7}$ (Hopf map) generators $[9,11]$ of the twistor string and its fermionic companion. They have a second order (covariant) bosonic reducibility, that reduces the number of independent $T$ 's to 7 . The whole set of constraints can be seen as defining the "twistor transform" between the different forms of superparticle dynamics.

The non-vanishing Poisson brackets between constraints are

$$
\begin{aligned}
& \left\{\pi_{\alpha}, \pi_{\beta}\right\}=2 \gamma_{\mu \alpha \beta} \Pi^{\mu}, \\
& \left\{T^{\alpha}, \pi_{\beta}\right\}=-2 \delta_{\beta}^{\alpha} t, \\
& \left\{T^{\alpha}, T^{\beta}\right\}=-\lambda^{\alpha} T^{\beta}+\lambda^{\beta} T^{\alpha}=\frac{1}{48} \gamma_{\mu \nu \rho}^{\alpha \beta}\left(\lambda \gamma^{\mu \nu \rho} T\right), \\
& \left\{T^{\alpha}, t\right\}=-\lambda^{\alpha} t .
\end{aligned}
$$

and one notices that $\Pi$ and $\pi$ generate an ordinary $D=10$ supersymmetry algebra, that has now become a gauge symmetry.

It is now straightforward to partially fix the gauge in different ways to arrive at the various advertised formulations: 
M. Cederwall, "A Note on the Relation between Different Forms of Superparticle Dynamics"

$i$. Use $\Pi$ to eliminate $(X, P)$ and $\pi$ to eliminate $(\vartheta, p)$. This gives the twistor formulation.

ii. Use $T$ and $\Pi$ (except $P^{2}=0$ ) to eliminate $(\lambda, \omega)$ and $t$ and part of $\pi$ to eliminate $\xi$. This gives the CBS superparticle (the fermionic second class constraints arise via Dirac brackets when the whole of $\pi$ is retained).

iii. Use $T$ and $\Pi$ (except $\left.P^{2}=0\right)$ to eliminate $(\lambda, \omega)$ and $\pi$ to eliminate $(\vartheta, p)$. This gives the spinning particle.

There is of course classes of formulations not reached through this procedure. These are the models of reference [7], where the twistor variables are multicomponent [14], and not of the simple division algebra type, and the ones using harmonic variables [15].

It is likely that this work has a connection to a not yet formulated $N=8$ supersymmetric formalism based on $S^{7}$. It is also plausible that extended supersymmetric field theories in $D=10$ may be given an off-shell symmetric formulation using the set of variables of this note.

\section{REFERENCES}

1. D.P. Sorokin, V.I. Tkach, D.V. Volkov and A.A. Zheltukhin, Phys.Lett. 216B (1989) 302.

2. R. Casalbuoni, Nuovo Cim. 33A (1976) 389;

L. Brink and J.H. Schwarz, Phys.Lett. 100B (1981) 310.

3. L. Brink, S. Deser, B. Zumino, P. Di Vecchia and P. Howe, Phys.Lett. 64B (1976) 435.

4. W. Siegel, Phys.Lett. 128B (1983) 397, Class.Quant.Grav. 2 (1985) 195.

5. I. Bengtsson and M. Cederwall, Göteborg-ITP-84-21 (1984).

6. D.P. Sorokin, V.I. Tkach and D.V. Volkov, Mod.Phys.Lett. A4 (1989) 901;

F. Delduc and E. Sokatchev, Class.Quant.Grav. 9 (1992) 361.

7. A. Galperin, P.S. Howe and K.S. Stelle, Nucl.Phys. B368 (1992) 248;

A. Galperin and E. Sokatchev, Phys.Rev. D46 (1992) 714.

8. I. Bengtsson Class.Quantum Grav. 4 (1987) 1143;

A.K.H. Bengtsson, I. Bengtsson, M. Cederwall and N. Linden, Phys.Rev. D36 (1987) 1766;

I. Bengtsson and M. Cederwall, Nucl.Phys. B302 (1988) 81;

M. Cederwall, Phys.Lett. 210B (1988) 169.

9. N. Berkovits, Phys.Lett. 247B (1990) 45;

M. Cederwall J.Math.Phys. 33 (1992) 388.

10. F. Englert, A. Sevrin, W. Troost, A. Van Proyen and Ph. Spindel, J.Math.Phys 29 (1988) 281.

11. M. Cederwall and C.R. Preitschopf, Göteborg-ITP-93-34, hep-th/9309030

12. L. Brink, M. Cederwall and C.R. Preitschopf, Phys.Lett. B311 (1993) 76.

13. Y. Eisenberg and S. Solomon, Nucl.Phys. B309 (1988) 709.

14. M. Cederwall, Göteborg-ITP-91-26.

15. A. Galperin, E. Ivanov, S. Kalizin, V. Ogievetsky and E. Sokatchev, Class.Quant.Grav. 1 (1984) 469, Class.Quant.Grav. 2 (1985) 155;

E. Sokatchev, Phys.Lett. 169B (1986) 209;

E. Nissimov, S. Pacheva and S. Solomon, Nucl.Phys. B296 (1988) 462, Nucl.Phys. B297 (1988) 349. 